\title{
February 2016 Imaging Case of the Month
}

\author{
Michael B. Gotway, MD \\ Department of Radiology \\ Mayo Clinic Arizona \\ Scottsdale, AZ
}

Clinical History: A 78 year-old woman presented to her physician for routine care. Her past medical history included hyperlipidemia, hypothyroidism, gout, hypertension, and arthritis.

Although she was asymptomatic, screening frontal and lateral chest radiography (Figure 1) was performed.

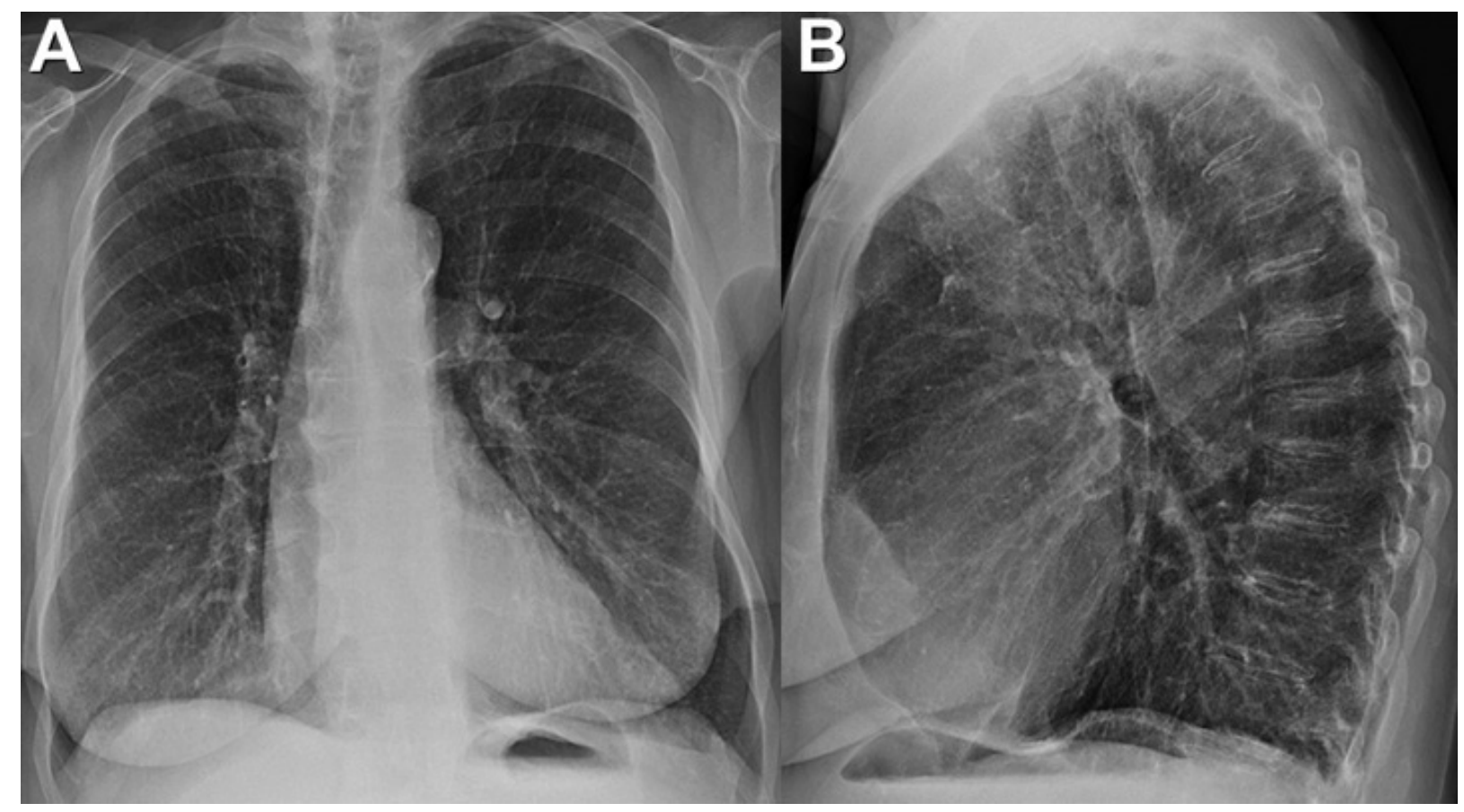

Figure 1. Frontal (A) and lateral (B) chest radiography.

Which of the following statements regarding the chest radiograph is most accurate?

1. The frontal chest radiograph shows a poorly defined nodular opacity in the left upper lung

2. The frontal chest radiograph shows abnormal mediastinal contours

3. The frontal chest radiograph shows basal predominant fibrotic abnormalities

4. The frontal chest radiograph shows large lung volumes with a cystic appearance

5. The frontal chest radiograph shows no abnormal findings 


\section{Correct! \\ 1. The frontal chest radiograph shows a poorly defined nodular opacity in the left upper lung}

The frontal and lateral chest radiograph shows fairly normal mediastinal contours, perhaps with a borderline heart size and tortuous aorta [not unexpected for age], with no evidence of cystic lung disease or fibrotic abnormalities. The chest radiograph is, however, abnormal; a faint nodular opacity is present in the left upper lung.

Which of the following is correct regarding the description of the chest radiographic findings of the left-sided lesion?

1. The left upper lobe nodular opacity demonstrates the "air crescent" sign

2. The left upper lobe nodular opacity shows evidence of calcification

3. The left upper lobe nodular opacity shows evidence of central cavitation

4. The left upper lobe nodular opacity shows intralesional fat consistent with hamartoma

5. The left upper lobe nodular opacity shows no specific morphological features 


\section{Correct! \\ 5. The left upper lobe nodular opacity shows no specific morphological features}

The left upper lobe nodular opacity is somewhat poorly defined, but ultimately shows no specific morphological features. In particular, no evidence of calcification or fat is present within the lesion. The "air crescent" sign is a specific form of cavitation within a nodule in which a thin, curvilinear rim of lucency, consistent with gas, partially surrounds a nodular intracavitary opacity, separating the nodule within the cavity from the wall of the cavity. The "air crescent" sign is typically encountered in the setting of angioinvasive fungal infection in immunocompromised patients, or, in the setting of relatively normal immunity, when fungal colonization of a pre-existing cavity occurs [mycetoma]. The presence of fat within a nodule is characteristic of hamartoma, but requires cross sectional imaging [typically thoracic CT] for demonstration; the small amounts of fat occurring with hamartoma would not be detectable with chest radiography.

The patient underwent unenhanced thoracic CT for further evaluation of the chest radiographic abnormality (Figure 2 ).

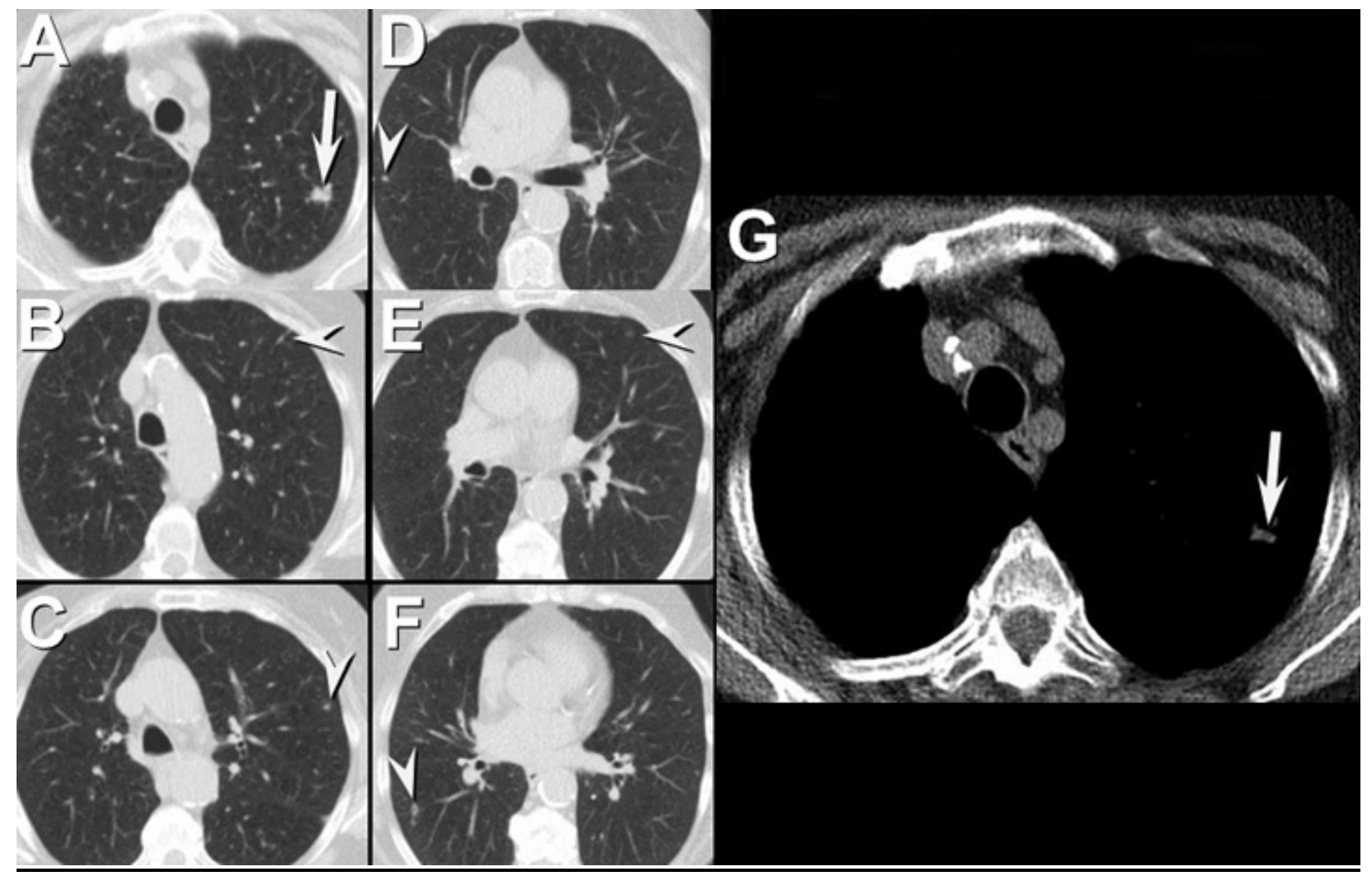

Figure 2. Axial unenhanced thoracic CT displayed in lung (A-F) and soft tissue (G) windows 
Regarding the thoracic CT examination, which of the following is correct?

1. The thoracic CT shows a non-specific, non-calcified, lobulated left upper lobe nodule corresponding to the chest radiographic finding

2. The thoracic CT shows fat within the left upper lobe nodule

3. The thoracic CT shows no abnormality corresponding to the chest radiographic finding, suggesting that the chest radiographic finding is artefactual

4. The thoracic CT shows numerous small nodules bilaterally consistent with a miliary pattern

5. The thoracic CT shows that the opacity corresponding to the chest radiographic abnormality has a morphology consistent with an arteriovenous malformation 


\section{Correct! \\ 1. The thoracic CT shows a non-specific, non-calcified, lobulated left upper lobe nodule corresponding to the chest radiographic finding}

The unenhanced thoracic CT shows a lobulated, non-calcified nodule in the left upper lobe, corresponding with the finding present at chest radiography. The CT shows no morphologically specific features, such as fat or calcium, which would suggest a specific diagnosis or allow the lesion to be regarded as likely benign. There is no evidence of a "feeding artery" or "draining vein" that would indicate that he nodule represents an arteriovenous malformation. A few other nodules are visible in the lungs bilaterally, but a "miliary" pattern- diffuse, randomly distributed small, round, discrete nodules (1-3 mm), and typical of a hematogenously disseminated process- is not present.

Which of the following represents an appropriate step for the evaluation of this patient?
1. ${ }^{18}$ FDG-PET scanning
2. ${ }^{68} \mathrm{Ga}$-citrate scanning
3. ${ }^{99 m}$ Tc-MAA ventilation-perfusion scintigraphy
4. Comparison to prior imaging
5. Repeat enhanced thoracic CT 


\section{Correct! \\ 4. Comparison to prior imaging}

When an indeterminate nodule is encountered at thoracic imaging, comparison to prior studies is almost always the single most important step that can be taken to establish the significance of the nodule. If comparison to prior studies, especially studies more than 2 years prior to the current examination, show that the nodule is present and unchanged, in most cases, the nodule may be regarded as benign, and an expensive evaluation, with its attendant patient-related anxiety and associated costs, may be avoided. In certain circumstances, particularly with subsolid (ground-glass opacity) nodules, the possibility of malignancy is not entirely excluded; however, even in this case, typically serial CT surveillance for possible nodule growth is all that is required for evaluation. Repeat contrast-enhanced thoracic CT would probably add little further information, and therefore the expense and potential risk associated with exposure related to the intravenous injection of iodinated contrast media is not justified. ${ }^{68} \mathrm{Ga}$ citrate scintigraphy is occasionally used for assessment of diffuse lung opacities, perhaps to detect opportunistic infections in immunocompromised patients, or pneumonitis related to medication-induced pulmonary injury, but none of these considerations are relevant to this patient. ${ }^{99 \mathrm{~m}} \mathrm{TC}-\mathrm{MAA}$ ventilation-perfusion scintigraphy is useful for the detection of suspected thromboembolic disease but pulmonary embolism is not highly suspected in this patient as the morphology of the left upper lobe nodule is not suggestive of an infarct; therefore ${ }^{99 \mathrm{~m}}$ Tc-MAA ventilation-perfusion scintigraphy is not the most appropriate next step. ${ }^{18}$ FDG-PET scanning is an appropriate modality for the further investigation of this patient's left upper lobe nodule, but the expense and radiation exposure associated with ${ }^{18}$ FDG-PET scanning should only be considered if prior studies are not available to assess the potential stability of the nodule retrospectively.

The patient indicated she had never undergone thoracic imaging previously, so ${ }^{18}$ FDGPET scanning (Figure 3) was performed. 


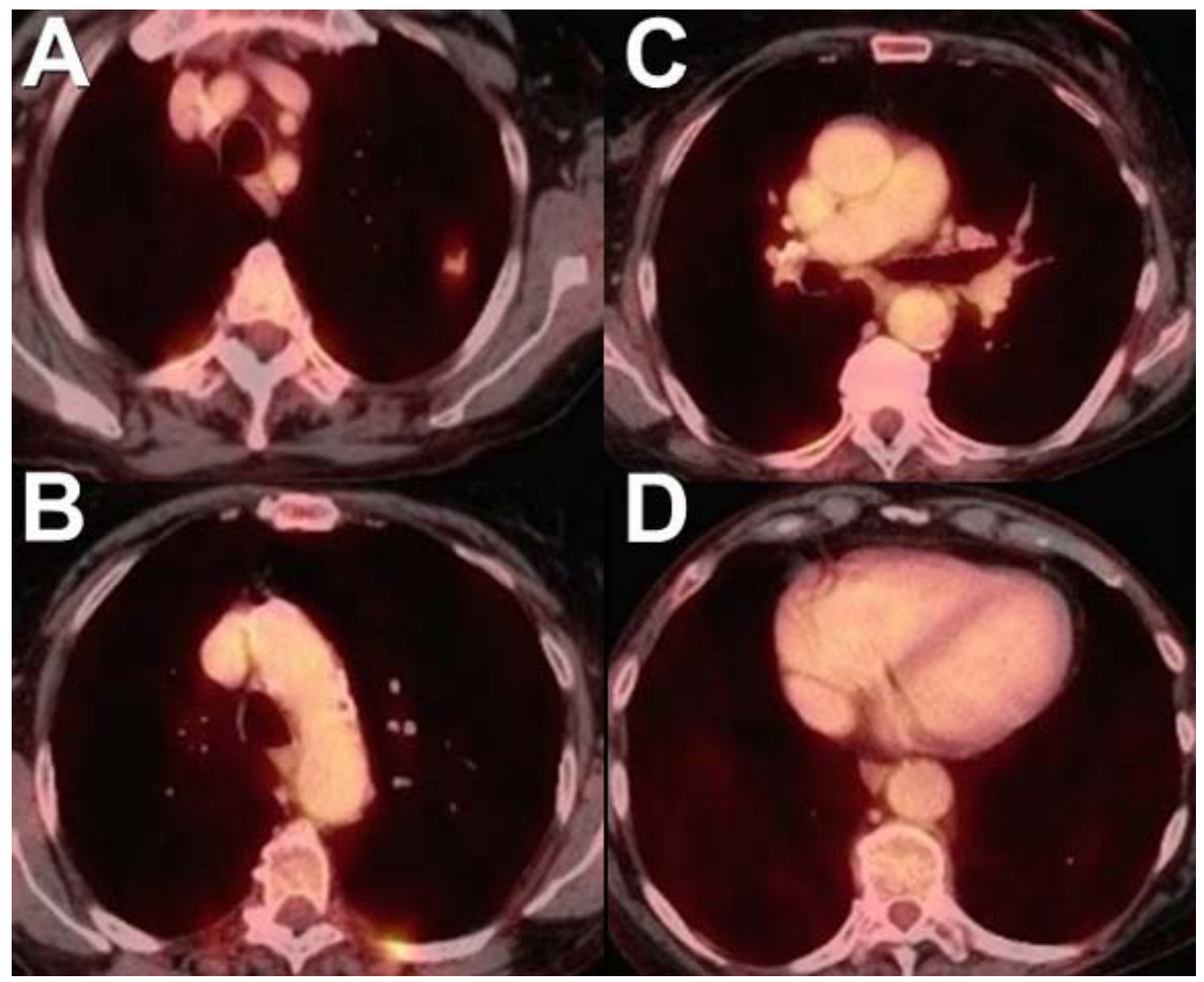

Figure 3. Selected ${ }^{18 F}$ FDG-PET CT images.

Regarding this examination, which of the following is correct?

1. The ${ }^{18}$ FDG-PET scan shows elevated tracer activity within peribronchial and mediastinal lymph nodes

2. The ${ }^{18}$ FDG-PET scan shows increased tracer activity within the left upper lobe nodules as well as other nodules

3. The ${ }^{18}$ FDG-PET scan shows increased tracer activity within the left upper lobe nodule only

4. The ${ }^{18}$ FDG-PET scan shows no metabolic activity within the left upper lobe nodule

5. The ${ }^{18}$ FDG-PET scan suffers from severe artefact- presumably from hyperglycemia or tracer mis-administration- and therefore provides no useful information 


\section{Correct! \\ 3. The 18FDG-PET scan shows increased tracer activity within the left upper lobe nodule only}

The ${ }^{18}$ FDG-PET scan shows increased tracer activity only within the left upper lobe; the other small nodules seen at thoracic CT show no tracer utilization. Note that these other nodules are quite small, and the lack of tracer utilization in such small nodules should not be taken as evidence that they are benign or non-aggressive in nature; rather, the nodules may simply be below the limit of ${ }^{18}$ FDG-PET scan resolution. No evidence of tracer utilization within mediastinal or peribronchial lymph nodes is noted on the images provided. The ${ }^{18}$ FDG-PET scan appears technically adequate- there is clear activity within blood pool, indicating that tracer has been properly administered and distributed.).

Based on the data thus far, which of the following represents the next most appropriate step for the evaluation of this patient?

1. Contrast-enhanced thoracic MRI

2. Flexible fiberoptic bronchoscopy

3. Open thoracotomy

4. Percutaneous transthoracic needle biopsy

5. Serial thoracic CT evaluation 


\section{Correct! \\ 4. Percutaneous transthoracic needle biopsy}

Among the choices provided, percutaneous transthoracic needle biopsy is the best choice to provide a specific diagnosis for this patient. Flexible fiberoptic bronchoscopy, particularly using navigational techniques, could potentially allow sampling of the lesion, but the lesion's small size and relatively peripheral nature suggest that flexible fiberoptic bronchoscopy would not be the best choice. Contrast-enhanced thoracic MRI would not add any useful information to that already available, and, in general, thoracic MRI is not rewarding for the evaluation of small pulmonary lesions. Open thoracotomy would certainly allow a definitive diagnosis, but remains needlessly aggressive at this stage. Note, however, given that several factors suggest a high pre-test probability for primary lung malignancy (morphology of the lesion, and the elevated tracer utilization at ${ }^{18}$ FDGPET scan), a reasonable argument could be made to proceed directly to lung resection on the assumption that any non-neoplastic results obtained by less invasive methods could represent false negative results. Nevertheless, it is common practice to obtain a tissue diagnosis for indeterminate pulmonary nodules before proceeding to surgical resection. Serial thoracic CT is often chosen for the evaluation of small nodules to assess for growth and is reasonable, even in this circumstance. However, given the aforementioned risk factors suggesting a high pre-test probability for malignancy for this nodule, there is a substantial possibility that the nodule is malignant, and therefore definitive action, rather than serial evaluation, is probably the best choice.

The patient underwent percutaneous transthoracic fine needle aspiration and core biopsy which was non-diagnostic.

Which of the following now represents the next most appropriate step for the evaluation for this patient?

1. Flexible fiberoptic bronchoscopy

2. Open thoracotomy

3. Repeat percutaneous transthoracic needle biopsy

4. Serial thoracic CT evaluation

5. Video-assisted thoracoscopic resection 


\section{Correct! \\ 5. Video-assisted thoracoscopic resection}

While a percutaneous transthoracic needle biopsy could reasonably be repeated, typically when this procedure fails to obtain satisfactory tissue for diagnosis, particularly when the needle is appropriately placed, it is appropriate to escalate to more invasive tissue sampling methods. In this case, open thoracotomy is again a reasonable consideration, but video-assisted thoracoscopic surgery provides a less invasive and typically less costly, approach for selected indeterminate pulmonary nodules. If a diagnosis of primary malignancy is obtained at video-assisted thoracoscopic surgical resection, often the procedure is then converted to an open thoracotomy to allow for formal lobectomy with lymph node sampling to provide definitive surgical therapy for cancer.

The patient underwent video-assisted thoracoscopic wedge resection of the left upper lobe nodule. The histopathological specimen is displayed in Figure 4.

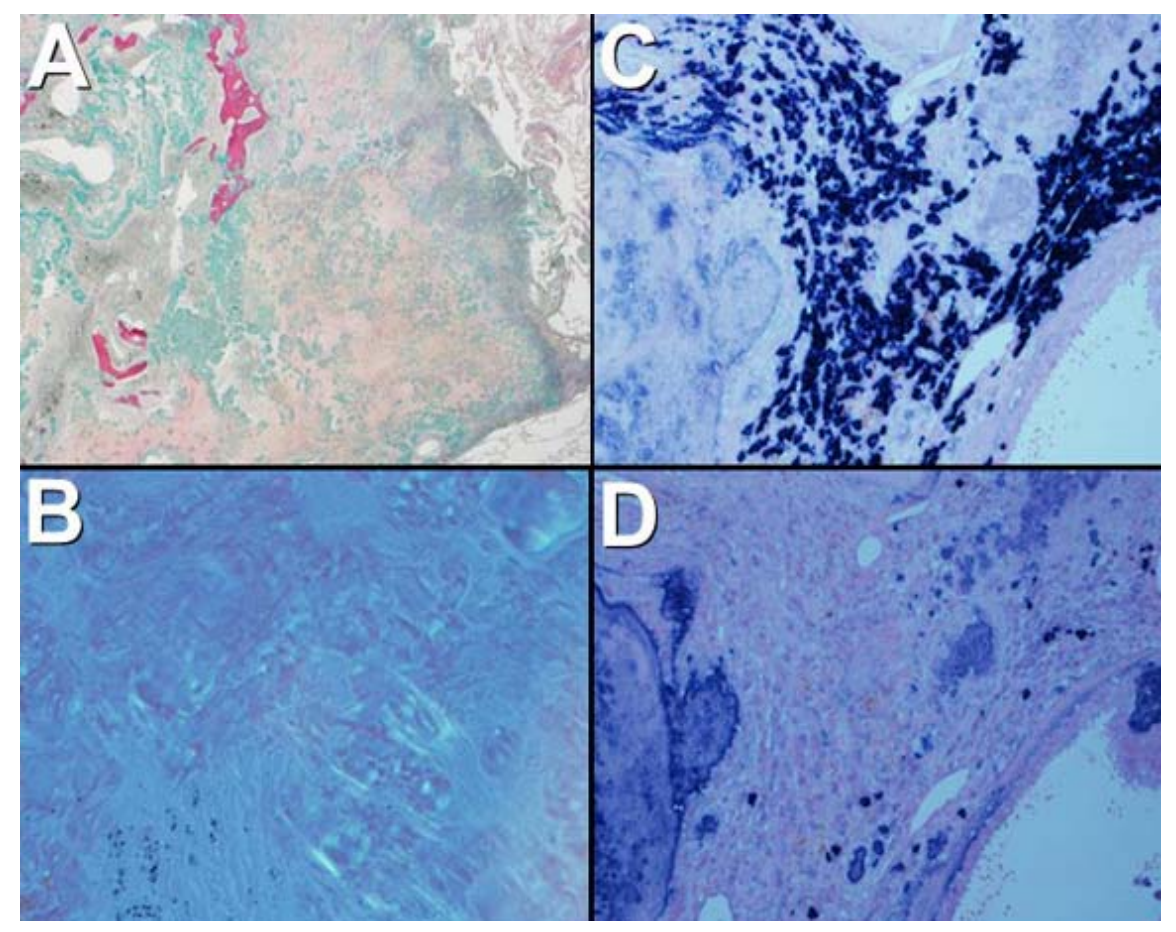

Figure 4. Histopathological specimen: $A=$ Sulfated alcian blue stain; $B=$ Congo red stain with polarized light; $C=$ kappa light chain stain; $D=$ lambda light chain stain.

Which of the following represents the diagnosis for this patient?

1. Amyloidosis

2. Bronchogenic carcinoma

3. Coccidioidomycosis

4. Hamartoma

5. Inflammatory myofibroblastic tumor 


\section{Correct! \\ 1. Amyloidosis}

The constellation of histopathological findings are consistent with the diagnosis of amyloidosis.

Diagnosis: Amyloidosis: AL (kappa light chain) - type associated with extranodal marginal zone lymphoma of mucosa-associated lymphoid tissue (MALT)

\section{References}

1. Algın O, Gökalp G, Topal U. Signs in chest imaging. Diagn Interv Radiol. 2011;17(1):18-29. [CrossRef] [PubMed]

2. Hansell DM, Bankier AA, MacMahon H, McLoud TC, Müller NL, Remy J. Fleischner Society: glossary of terms for thoracic imaging. Radiology. 2008;246(3):697-722. [CrossRef] [PubMed]

3. Lantuejoul S, Moulai N, Quetant S, Brichon PY, Brambilla C, Brambilla E, Ferretti GR. Unusual cystic presentation of pulmonary nodular amyloidosis associated with MALT-type lymphoma. Eur Respir J. 2007;30(3):589-592. [CrossRef] [PubMed]

4. Jeong YJ, Lee KS, Chung MP, Han J, Chung MJ, Kim KI, Seo JB, Franquet T. Amyloidosis and lymphoproliferative disease in Sjögren syndrome: thin-section computed tomography findings and histopathologic comparisons. J Comput Assist Tomogr. 2004;28(6):776-781. [CrossRef] [PubMed]

5. Lee AY, Godwin JD, Pipavath SN. Case 182: pulmonary amyloidosis. Radiology. 2012;263(3):929-932. [CrossRef] [PubMed] 